\title{
Preparation and characterization of albumin nanoparticles encapsulating curcumin intended for the treatment of breast cancer
}

\author{
AV Jithan, K Madhavi ${ }^{1}$, M Madhavi ${ }^{2}$, K Prabhakar ${ }^{3}$ \\ Department of Pharmaceutics, Mother Teresa College of Pharmacy, Ghatkesar, Hyderabad, ${ }^{1}$ Care College of Pharmacy, ${ }^{2}$ Vagdevi College of \\ Pharmacy, ${ }^{3}$ University College of Pharmaceutical Sciences, Kakatiya University, Warangal - 506009 , India.
}

\begin{abstract}
Introduction: For the real-time clinical utilization of curcumin (an ayurvedic natural product) to treat breast cancer, its dissolution, rate limited solubility, poor tissue absorption, and extensive in vivo metabolism that leads to its poor systemic bioavailability should be overcome. A polymer-based nanoparticle formulation using bovine serum albumin can increase its aqueous solubility and can achieve protected, sustained, and targeted therapy in breast cancer. Materials and Methods: Desolvation technique was optimized for the preparation of albumin nanoparticles. Particle size, drug release, encapsulation efficiency, drug polymer interaction were the in vitro properties that were determined. Cell culture studies, in vivo pharmacokinetics in rats were used for biological characterization of the formulation. Results: The formulations were successfully prepared using $1: 1,1: 2,1: 3,1: 4$ drug: polymer ratios and the percent entrapment was found to be $74.76 \%, 91.01 \%, 85.36 \%, 86.42 \%$, respectively, and particle size determined by zetasizer was found to be $225.1,223.5,226.3,228.7 \mathrm{~nm}$, respectively, and in vitro release was sustained for at least one month with drug release of $75.74 \%, 65.97 \%, 64.42 \%, 54 \%$, respectively. The dissolution rate and aqueous solubility of curcumin was enhanced with this formulation. Fourier transform infrared spectroscopy (FTIR) studies demonstrated that the drug was not changed in the formulation during the fabrication process. The proliferation assays in MDA-MB-231 tumor cell lines indicated more effectiveness of the formulation compared to its solution form. In rats, albumin nanoparticles sustained drug release, demonstrated more bioavailability, improved pharmacokinetic properties, and enhanced tissue targetability of the drug. Conclusions: An effective curcumin-albumin nanoparticle formulation was successfully developed using a desolvation technique.
\end{abstract}

Key words: Albumin nanoparticles, breast cancer, curcumin, solubility, sustained release

\section{INTRODUCTION}

Curcumin is a natural product that possesses several pharmacological properties. Especially, it has been demonstrated to be a superior anticancer agent against several types. ${ }^{[1]}$ These activities have been demonstrated after parenteral or oral

\section{Address for correspondence:}

Dr. AV Jithan,

Mother Teresa College of Pharmacy,

NFC Nagar, Ghatkesar, Hyderabad, India.

E-mail: aukunjv@gmail.com

\begin{tabular}{|l|l|}
\hline \multicolumn{2}{|c|}{ Access this article online } \\
\hline Quick Response Code: & Website: \\
\hline & www.jpionline.org \\
\cline { 2 - 2 } & \\
\hline & \\
\hline
\end{tabular}

administrations in animal models or using in vitro assays. ${ }^{[2]}$ In animals, curcumin reduced carcinogen-induced tumor genesis and inhibited growth of implanted human tumors. ${ }^{[2,3]}$ Such studies have led to clinical trials of curcumin in patients with colon cancer. ${ }^{[4]}$ Unfortunately, the study along with several other preclinical trials has revealed that one of the major problems with curcumin is its poor bioavailability in vivo after any route of administration. However, because it holds significant therapeutic promise, its clinical potential is being investigated by various groups. ${ }^{[4-8]}$ Several drug delivery issues needs to be addressed for rediscovering the practical utility of this molecule. Its dissolution rate limited solubility in aqueous solvents is the major problem. It has poor oral bioavailability and undergoes extensive systemic metabolism. These three rate-limiting factors together leads to less effectiveness of this molecule in clinics, particularly its systemic bioavailability is compromised.

Several drug delivery strategies demonstrated enhancement in the in vitro and in vivo properties of curcumin by altering 
the weaknesses of the intrinsic properties of this drug. Solid dispersions successfully improved curcumin's solubility and dissolution in simulated gastric fluids. ${ }^{[9]}$ Cyclodextrin complexation also demonstrated better solubility for curcumin and after oral administration this complex demonstrated better anti-inflammatory activity in rat colitis model compared to pure curcumin. ${ }^{[10]}$ Several other such examples have been published. ${ }^{[1-15]}$ Previously biodegradable polymers were used to prepare curcumin nanoparticles and demonstrated improvement in solubility and hence pharmacological activities. ${ }^{[16,17]}$ However, among these protein-based nanoparticle carrier systems utilizing serum albumins only one report was very recently published as per our knowledge and this formulation represent a very attractive strategy. Kim et al., recently developed curcuminalbumin nanoparticles using albumin bound technology ${ }^{[17]}$ and not the desolvation technique we used in this study. Albumin nanoparticles are biodegradable, non-antigenic, and relatively easy to prepare. ${ }^{[18]}$ Curcumin nanoparticles with albumin combines high drug loading capacity with minimal tissue irritation and toxic effects. Also, with serum albumin nanoparticles, significant amount of drug can be incorporated into the particle matrix because of the different drug-binding sites present in the albumin molecule. A substantial binding of administered curcumin to serum albumin was noted ${ }^{[19]}$ Previously, it was shown that albumin nanoparticles enhanced endocytic uptake of drugs. ${ }^{[20]}$ Thus, intracellular levels also can be enhanced with the curcumin nanoparticle formulation. Further, it was noted that because of the EPR effect albumin nanoparticles of sizes approximately $\sim 200 \mathrm{~nm}$ are retained in the tumor vasculature. The successful endocytic cellular uptake along with successful tumor retention of albumin nanoparticles leads to a successful passive targeting of drugs to tumors with this delivery system. ${ }^{[21]}$ Also, albumin nanoparticles are known to increase the solubility of poorly soluble drugs used for i.v. administration, and thus the toxic solubilizers such as cremophor used for the same reason can be avoided. ${ }^{[17]}$ In fact, a nanoparticle formulation of paclitaxel in which serum albumin is included as a carrier (Abraxane) has been approved for the treatment of breast cancer. $^{[21]}$

Main objective of this study was to prepare curcumin-albumin nanoparticles using a desolvation technique. Further, the objective was to evaluate this novel intravenous curcumin nanoparticular formulation for sustained drug release, targeted and enhanced intracellular drug release and enhancement in bioavailability.

\section{MATERIALS AND METHODS}

Curcumin (minimum 94\% curcuminoid content) was purchased from Yarrow enterprises, Mumbai. Bovine serum albumin [fraction v] specification - Albumin min 98.5\%, was purchased from Qualikems fine chem. Pvt. Ltd, Mumbai. Glutaraldehyde was purchased from Finar chemicals limited, Ahmadabad. Methanol was purchased from Merck chemicals limited.
Methanol HPLC grade was purchased from Merck specialties, New Delhi. Ethyl acetate was purchased from Qualikems Fine chem. All the other chemicals were of analytical grade. Magnetic stirrer, cyclomixer, ultracentrifuge and micro centrifuge bought from REMI Equipments Pvt. Ltd. were used. HPLC from Cyber Labs, USA was used in the analysis of drug levels in the plasma and the tissues. A Shimadzu UV-Visible spectrophotometer was used in the analysis of samples from in vitro drug release and drug content assays. A bath sonicator from Pci Equipments was used. Male Wister rats (weighing 150-180 g) were purchased from Mahaveer enterprises, Hyderabad. The experimental protocol was approved by Institutional Animal Ethical Committee (Registration No: 1047/ac/07/CPCSEA) constituted in accordance with the rules and guidelines of the committee for the purpose of control and supervision of experiments on animals (CPCSEA), India. All the surgicals used in this study were sterile and procured from standard sources.

\section{Preparation of curcumin-albumin nanoparticles and their in vitro characterization}

Curcumin nanoparticles were prepared using biodegradable bovine serum albumin as the polymer using a desolvation technique. The protocol followed was a previously published method. ${ }^{[22]}$ Briefly, bovine serum albumin between $50 \mathrm{mg}$ and $200 \mathrm{mg}$ was dissolved in $2.0 \mathrm{ml}$ of purified distilled water. Subsequently, curcumin was dissolved in $8 \mathrm{ml}$ ethanol, which was added drop wise into the aqueous albumin solution under magnetic stirring $(500 \mathrm{rpm})$. This resulted in the formation of an opalescent suspension spontaneously at room temperature. After this desolvation process, $0.11 \mathrm{ml}$ of $8 \%$ glutaraldehyde in water $(\mathrm{v} / \mathrm{v})$ was added to cross-link the desolvated bovine serum albumin nanoparticles. The cross linking process was performed under stirring of the colloidal suspension over a time period of 24 hours. The resulting suspension was purified by 5 cycles of differential centrifugation $\left(12,000^{*} \mathrm{~g}, 8 \mathrm{~min}\right)$ and redispersed the pellet to the original volume in distilled water. Each redispersion step was performed in a bath sonicator over 5 mins. The formation of nanoparticles using this technique was confirmed by a scanning electron microscope and a zetasizer.

The formation of nanoparticles, their size and morphology were examined using scanning electron microscopy (SEM). Values of effective hydrodynamic diameter in dilute dispersions were obtained by dynamic light scattering measurements using a zeta sizer (ZetaPlus zeta potential analyzer, Brookhaven Instruments Corp, Holtsville, NY, USA) equipped with a laser source at a fixed angle of $90^{\circ}$. Prior to analysis, the solutions were filtered through a filter (pore size $\approx 2 \mu \mathrm{m}$ ) to remove dust. Each sample was measured in triplicate. After their fabrication, the percent yield, entrapment efficiency, drug release and enhancement in drug solubility, the drug-polymer interaction were evaluated. To determine the percent yield, the nanoparticle formulation was centrifuged at the end of preparation, the debris were removed, washed, and centrifuged twice to obtain a pellet. This pellet of nanoparticles was weighed and the percent yield was calculated using the following equation. 
Percent yield $=\frac{\text { Weight of the nanoparticles }}{\text { Total weight of drug }+ \text { polymer }} \times 100$

For determination of drug entrapment, the amount of drug present in the supernatant (obtained at the end of preparation of nanoparticles) after centrifugation was determined (w) by UVspectrophotometer. A standard calibration curve of concentration vs. absorbance was plotted for this purpose. The amount of drug in supernatant was then subtracted from the total amount of drug added during the desolvation process $(\mathrm{W})$. ( $\mathrm{W}-\mathrm{w})$ will give the amount of drug entrapped in the pellet. Then exact percentage entrapment is given by $(W-W) / W * 100$.

The in vitro drug release profiles of albumin nanoparticles were determined as follows. These experiments were conducted in dark conditions as curcumin extensively degrades in the presence of light. The release medium was phosphate-buffered saline (PBS, pH 7.4), which contained ascorbic acid at a concentration of $1 \%$ and butylated hydroxytoluene at a concentration of $0.1 \%$ to prevent further degradation. Although it was previously reported that stability in PBS was very less for curcumin, our release studies indicated that the methodology we employed for the study did not lead to any significant degradation during the study period with aqueous solubilized curcumin in PBS. Thus, this method can be conveniently used to study the release of the curcumin from a variety of formulations. About $100 \mathrm{mg}$ nanoparticles were redispersed in $200 \mathrm{ml}$ of the release medium. The entire system was kept at $(37 \pm 0.5)^{\circ} \mathrm{C}$ under stirring at $100 \mathrm{rpm}$. At designed time intervals [30 min, 1, 2, 3, 4, 5, 6, $12 \mathrm{hrs}$ and 1, 2, 3 till 30 days], $20 \mathrm{ml}$ of the release medium was removed and replaced with the same volume of fresh medium. The samples withdrawn were filtered using $0.2 \mu$ sterile filter and the amount of curcumin in the release medium was determined by UV-spectrophotometer at $425 \mathrm{~nm}$. All measurements were performed in triplicate. The cumulative amount of drug release over the time period was plotted. The data was fitted to various models that indicate the type of drug release. To determine the enhancement in the solubility with the formulation, solubility studies with curcumin and with the formulations were conducted. These experiments were also conducted in dark conditions. Briefly, an accurately weighed amount $(100 \mathrm{mg}$ ) of nanoparticles was added to $500 \mathrm{ml}$ of release medium. The solubility of free curcumin and curcumin from the nanoparticles were investigated using the USP rotating paddle dissolution apparatus at $100 \mathrm{rpm}$ and $37 \pm 0.5^{\circ} \mathrm{C}$. Five milliliter of samples were withdrawn from the dissolution medium at various time intervals $30 \mathrm{mins}, 2,4$, 6,8 , and $24 \mathrm{hrs}$, replaced with fresh dissolution medium. The withdrawn samples were filtered using $0.2 \mu$ sterile filter and analyzed by UV-spectrophotometer. All solubility studies were performed in triplicate.

Drug-polymer interactions was investigated using Fourier Transform Infrared (FTIR). FTIR of drug (curcumin), polymer (bovine serum albumin), placebo nanoparticles, and drug-loaded nanoparticles were taken using a Thermo Nicolet Nexus 670 spectrophotometer with $\mathrm{KBr}$ pellets. The sample was prepared and analyzed under general conditions ( $\mathrm{pH}$ value of 7 , temperature $24^{\circ} \mathrm{C}, 200 \mathrm{rpm}$ agitation).

\section{Biological characterization of curcumin-albumin}

The biological characterization of CA nanoparticles was performed by determining the sustained in vivo drug release and tissue levels in the male rats as well as determining the cell viability assay in breast adenoma carcinoma. Intracellular drug levels and the activity were assessed based on the cell viability.

In vivo drug release and tissue uptake studies were conducted after intravenous administration of the formulation and comparing the standards. Different curcumin formulations were prepared and include:

1. Curcumin solution: Solutions of curcumin $(5 \mathrm{mg} / \mathrm{ml}$ and $10 \mathrm{mg} / \mathrm{ml}$ ) for intravenous administration to the rats was prepared by dissolving the drug in $0.5 \mathrm{ml}$ of a $50: 50$ mixture of poly ethylene glycol (PEG) 400 and $95 \%$ ethanol.

2. CA nanoparticular suspension: CA nanoparticles (containing $10 \mathrm{mg}$ of the drug) were suspended in $0.5 \mathrm{ml}$ normal saline $(0.9 \% \mathrm{w} / \mathrm{v})$ for intravenous injection to rats.

The study was conducted using 12 wistar rats and they were divided into three groups

- Group 1: This group was given $10 \mathrm{mg}$ equivalent curcumin solution intravenously over a period of 30 days (daily dose: $330 \mu \mathrm{g})$.

- Group 2: This group was given $10 \mathrm{mg}$ equivalent curcumin solution intravenously on day 1.

- Group 3: This group was given CA nanoparticles of $10 \mathrm{mg}$ curcumin equivalent formulation intravenously on day one (single time administration).

To determine the plasma profile in the three groups, blood samples $(0.5 \mathrm{ml})$ were collected from retro orbital sinus of rat eye under anesthesia at intervals of 1, 3, 6, 12 and 24 hrs. For nanoparticular curcumin suspension system along with above time intervals, samples were also collected after 3, 6, 9, up to 30 days. The blood samples collected were added to micro centrifuge tubes containing $0.3 \mathrm{ml}$ of sodium citrate solution $(4 \% \mathrm{w} / \mathrm{v}$ in water). All the samples were centrifuged at $3000 \mathrm{rpm}$ for $10 \mathrm{mins}$ and plasma was separated into another micro centrifuge tube by using micro pipette and stored in deep freeze. The drug was extracted from the plasma by adding $500 \mu \mathrm{l}$ of ethyl acetate, and vortexed on cyclomixer for 20 mins. The organic phase was separated and collected into another micro centrifuge tube and allowed to air for $24 \mathrm{hrs}$ by keeping the lid of the tube open. These dried tubes were stored in deep freeze until HPLC analysis was performed. Before the HPLC analysis samples were reconstituted with $200 \mu$ l of mobile phase [methanol: buffer pH 3.0 (72:28)] and analyzed at $262 \mathrm{~nm}$ wavelength.

To determine the drug levels in the tissues, a previously published method was used. ${ }^{[23]}$ Briefly, after the experimental study was completed (after 30 days), all the rats from Group 1 and Group 3 were sacrificed. Then liver, lung, and brain were carefully isolated and washed. Tissue samples were separately homogenized in $2 \mathrm{ml}$ 
distilled water, lyophilized, and minced with $2 \mathrm{ml}$ of extracting agent ( $95 \%$ ethyl acetate $+5 \%$ methanol). The contents were centrifuged at $3000 \mathrm{rpm}$ for about 15 mins and the supernatant liquid was separated using micro pipette and taken in a fresh test tube and organic layer was evaporated to dryness. Before HPLC analysis, it was reconstituted with $200 \mu$ l of mobile phase [methanol: buffer pH 3.0 (72:28)] and analyzed at 262 nm wavelength.

The cell viability assay with curcumin-albumin nanoparticles was carried out in MDA-MB-231 (breast adenocarcinoma). The cells were cultured in Dulbecco's-modified eagles medium supplements with $1 \%$ Fetal Bovine Serum (FBS). Culture media were supplemented with $100 \mathrm{U} / \mathrm{ml}$ penicillin and 100 $\mu \mathrm{g} / \mathrm{ml}$ streptomycin at $37^{\circ} \mathrm{C}$ in a humidified atmosphere under $5 \% \mathrm{CO}_{2}$. Curcumin stock solution of $10 \mathrm{mM}$ was prepared by dissolving in dimethyl sulphoxide (DMSO) and the stock was diluted to the required concentration immediately before use and nanoparticular curcumin suspension of different concentrations were prepared using phosphate buffer of $\mathrm{pH} 7.4$, redispersed in a bath sonicator, and used. Cells grown in media containing equivalent amount of DMSO without curcumin served as control. Cell viability was assessed by MTT [(3, 4, 5-dimethyl thiazol-2 -yl)-5-5 diphenyl tetrazolium bromide] assay. After $24 \mathrm{hrs}$, the cells were treated with different concentrations of curcumin $(0,10,20,30,40 \mu \mathrm{m})$. After the $72 \mathrm{hr}$ treatment, media containing curcumin were carefully removed, cells were washed twice with PBS, before $100 \mu \mathrm{l}$ medium with $0.5 \mathrm{mg} / \mathrm{ml}$ MTT in PBS was added to each well. The plate was incubated at $37^{\circ} \mathrm{C}$ for $4 \mathrm{hrs}$. Then the medium was totally removed and $200 \mu \mathrm{l}$ TRIS-DMSO solution was added to each well. The plate was vibrated for 30 mins. The absorbance, which was proportional to cell viability, was subsequently measured at $570 \mathrm{~nm}$ in each well using an ELISA plate reader (Biopad). At least three independent experiments were performed and the same procedure was repeated for nanoparticular curcumin redispersed in PBS.

The $\%$ cell viablility $=$ Absorbance of treated cells Absorbance of control cells $\times 100$

Proliferation Inhibition ratio $(\%)=\frac{\mathrm{A}-\mathrm{B}}{\mathrm{A}} \times 100$

Where $\mathrm{A}$ is the absorbance of control cells and $\mathrm{B}$ is the absorbance of treated cells

\section{RESULTS AND DISCUSSION}

Curcumin is like an old wine in a new bottle. There is an interest in clinical development of this agent as an anti-cancer drug. It is attracting lot of scientists from India as well as the Western countries to do further research. Interestingly, recent publications clearly demonstrate that a new powerful formulation of this ayurvedic molecule donned in novel drug delivery systems such as nanoparticles will be in the market very soon. This formulation can be used in the treatment of several cancers. As a continuation to this interest, in this study an i.v. administered nanoparticular formulation encapsulating curcumin using albumin as the polymer was developed. This study particularly focussed on its utility in breast cancer. The pharmaceutical aim is to enhance its in vitro/in vivo solubility, protect it from systemic degradation, and increase its bioavailability. The confirmation of the nanoparticle formation using desolvation technique for curcumin with albumin was obtained using SEM [Figure 1] and zeta sizer [Table 1]. Curcumin nanoparticles were successfully prepared with different drug polymer ratio of 1:1, 1:2, 1:3, and 1:4. Albumin nanoparticles showed an average size of about 225.1, 223.5, 226.3, $228.7 \mathrm{~nm}$, respectively measured by zeta sizer. Thus it can be said that drug polymer ratio do not have a significant influence on the particle size. The entrapment efficiency found was 74.76, 91.01, $85.36,86.42$, respectively, observed by UV-spectrophotometer and percent yield was $51.9 \%, 84.3 \%, 85 \%, 85.2 \%$, respectively. The nanoparticles showed high zeta potential of $-32.2,-31.7,-30.1$, $-30.5 \mathrm{mV}$, respectively. The charge also did not vary significantly with the use of various ratios. Various factors for a successful

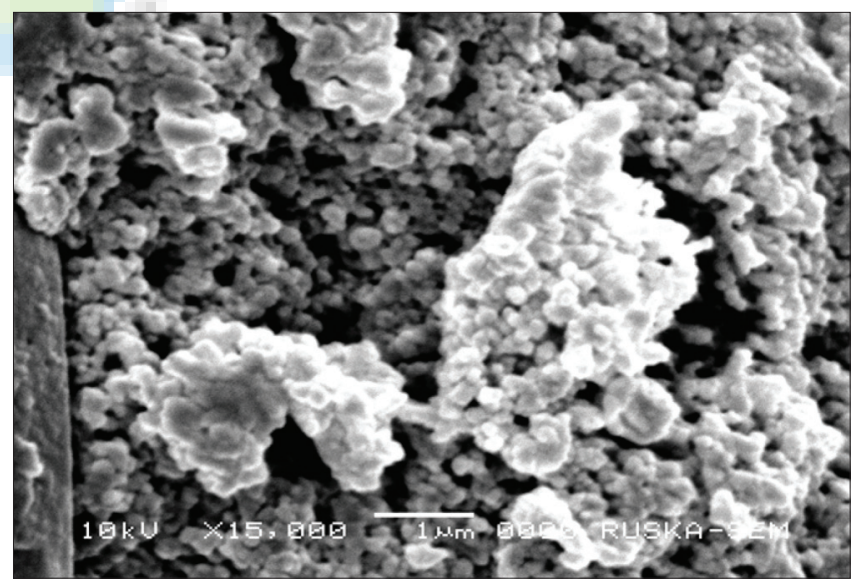

Figure 1: Scanning electron microscopy picture of albumin nanoparticles

\begin{tabular}{lcccc}
\multicolumn{2}{l}{ Table 1: Particle size, zeta potential, and entrapment efficiency of curcumin nanoparticles } \\
\hline Formulation code & $\begin{array}{c}\text { Particle size } \\
\text { (mean } \pm \text { SD) } \text { nm }^{*}\end{array}$ & $\begin{array}{c}\text { Polydispersity } \\
\text { Index (PDI) }\end{array}$ & $\begin{array}{c}\text { Zeta potential } \\
\text { (mean } \pm \text { SD) }\end{array}$ & \% Entrapment Efficiency(mean \pm SD)* \\
\hline CNP1 & $225.1 \pm 1.32$ & 0.120 & $-32.2 \pm 6.1$ & $74.76 \pm 3.45$ \\
CNP2 & $223.5 \pm 1.66$ & 0.141 & $-31.7 \pm 7.7$ & $91.01 \pm 2.15$ \\
CNP3 & $226.3 \pm 1.53$ & 0.238 & $-30.1 \pm 5.43$ & $85.36 \pm 1.24$ \\
CNP4 & $228.7 \pm 1.22$ & 0.364 & $-30.5 \pm 7.46$ & $86.42 \pm 0.75$ \\
\hline
\end{tabular}

*Values indicate mean \pm standard deviation (SD). $n=3$ 
nanoparticle formulation should be considered. Physicochemical characterization that influences the physical stability, cellular uptake, biodistribution and release of encapsulated drug in the plasma is essential after the nanoparticle is formulated. Smaller size nanoparticles $(\approx 200 \mathrm{~nm})$ are advantageous for passive targeting to tumor tissue by enhanced permeability and retention effect and higher zeta potential influence the particle stability, cellular uptake, and intracellular trafficking. Thus, the curcumin albumin nanoparticles developed here, which has a small and appropriate size and high surface charge could enhance circulation half lives, evades the reticulo-endothelial system (RES) and lead to enhanced tissue levels. Further, to confirm the presence of curcumin in albumin nanoparticles, FTIR analysis was considered. The FTIR reports of curcumin, bovine serum albumin, placebo nanoparticles, curcumin-albumin nanoparticles indicated that curcumin exists inside the albumin nanoparticles (data not shown). It also confirmed lack of any drug polymer interaction, which is indicated by the lack of additional peak in the FTIR for curcumin nanoparticles.

A biphasic release pattern of entrapped curcumin from albumin nanoparticles was observed in the release studies. Herein, a rapid release of about $27.75 \%, 23.98 \%, 23.68 \%$, $19.9 \%$, respectively from different formulations in $24 \mathrm{hrs}$ was observed, followed by a sustained first order drug release of about $75.74 \%, 65.98 \%, 64.42 \%, 54 \%$, respectively over 30 days of the experiment [Table 2]. The observed initial burst release might be due to the dissociation of surface absorbed drug present in the polymeric matrix. Subsequently, sustained release activity of the drug was due to the slow release of drug entrapped inside the polymeric matrix. Previous studies with other types of curcumin encapsulated nanoparticulates demonstrated similar results. ${ }^{[17]}$

The curcumin released from albumin nanoparticles dissolved in phosphate buffer $\mathrm{pH} 7.4$ was almost 4-fold higher than that of free curcumin [Figure 2]. The results suggest that the solubility of curcumin with curcumin nanoparticles can be enhanced in vivo, thereby promoting higher plasma levels as well as higher intracellular levels, and thus leading to a better therapeutic activity. This is reflected in higher peak concentrations in the plasma after $1 \mathrm{hr}$ of administration. Poor oral bioavailability of curcumin occurring most often limits its clinical use. In phase I clinical trial, oral administration of $3.6 \mathrm{~g}$ of curcumin daily resulted in $11.1 \mathrm{nmol} / \mathrm{L}$ plasma concentration. Other studies also indicated the same. This is true with any route of administration. This low plasma concentration is partly due to its low rate of dissolution in aqueous medium and significant metabolism in the systemic circulation. Shortly, some studies with a variety of polymers addressed this issue successfully. Our studies also aimed to increase the dissolution rate of the drug with the formulations. The results indicated our success in such an enhancement. Inherently, nanoparticle formulation protects the drugs from the systemic circulation. Thus, the enhancement in the dissolution rate successfully enhances the bioavailability of the drug in the systemic circulation and higher concentrations of drugs may be present in the target site with our formulation. This is evidenced by higher systemic drug levels with the nanoparticle formulation. However, in this regard further studies have to be conducted to further enhance the dissolution rate of solubility of curcumin so as to lead to higher bioavailabilities. In the study by Kim et al., ${ }^{[17]}$ the solubility was enhanced by 300 -fold. In their study, the solubility was enhanced from $2.792 \mu \mathrm{g} / \mathrm{ml}$ to $851.416 \mu \mathrm{g} / \mathrm{ml}$. On the other hand, in our study the solubility was found to increase from $0.6 \mu \mathrm{g} / \mathrm{ml}$ to $2.6 \mu \mathrm{g} / \mathrm{ml}$ (please note solubility values as determined in our laboratory using our solubility determination methodology). Thus, our formulation has to be enhanced for a further development leading to clinical utility of this ayurvedic therapeutic agent. Different approaches such as change in the polymer, studying different drug polymer ratios, modifying the fabrication process, etc. can be attempted.

Albumin nanoparticles were prepared to increase the bioavailability of delivered curcumin. In this view, the nanoparticles and free curcumin was administered $10 \mathrm{mg}$ intravenously to rats (Group 2 and Group 3). The mean curcumin concentration in the serum of rats after intravenous administration of both free curcumin and nanocurcumin were determined. Result showed

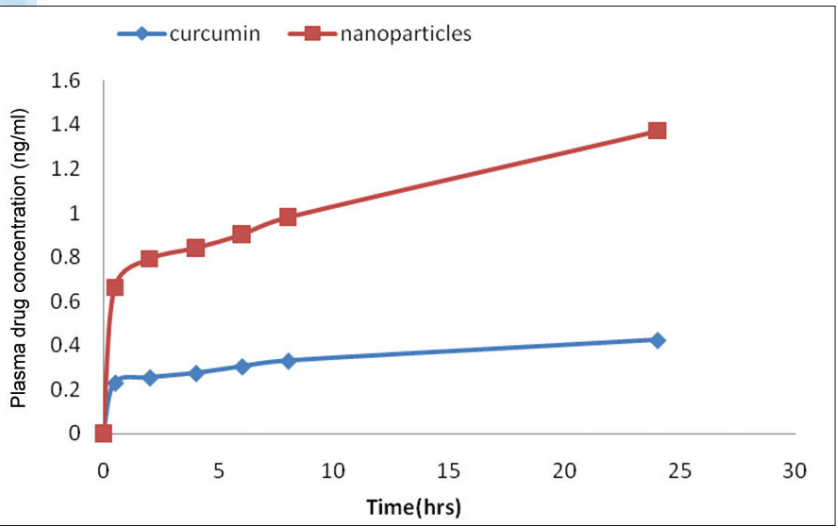

Figure 2: Solubility enhancement of curcumin with albumin nanoparticles

Table 2: Release model kinetics applied to curcumin nanoparticles

\begin{tabular}{|c|c|c|c|c|}
\hline \multirow[t]{2}{*}{ Formulation code } & Zero order & First order & Higuchi model & Korsemeyer-Peppas model \\
\hline & $\mathbf{R}^{2}$ & $\mathbf{R}^{2}$ & $\mathbf{R}^{2}$ & $\mathbf{R}^{2}$ \\
\hline CNP1 & 0.9781 & 0.9817 & 0.9688 & 0.9248 \\
\hline CNP2 & 0.9787 & 0.9877 & 0.9696 & 0.9238 \\
\hline CNP3 & 0.9777 & 0.9871 & 0.9695 & 0.9255 \\
\hline CNP4 & 0.9768 & 0.9874 & 0.9694 & 0.9268 \\
\hline
\end{tabular}


Table 3: Curcumin levels in various tissues (Mean \pm S.E.M; $\mathbf{n}=3$ )

\begin{tabular}{lcc}
\hline Tissue & $\begin{array}{c}\text { Tissue levels of curcumin after i.v. } \\
\text { solution administration }(\mathbf{n ~ m o l} / \mathbf{g})^{*}\end{array}$ & $\begin{array}{c}\text { Tissue levels of curcumin after i.v. administration } \\
\text { of curcumin nanoparticles }(\mathbf{n} \text { mol/g) }\end{array}$ \\
\hline Liver & $7.3 \pm 2$ & $23 \pm 6$ \\
Lung & $1.6 \pm 0.9$ & $4.6 \pm 1$ \\
Brain & $0.3 \pm 0.2$ & $0.9 \pm 0.6$ \\
\hline
\end{tabular}

*Values indicate mean \pm standard error mean (S.E.M). $n=3$

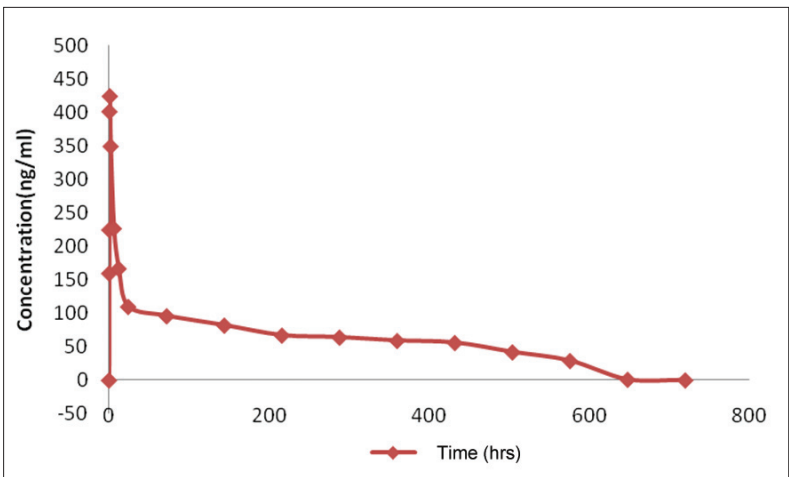

Figure 3: Plasma drug concentrations observed after administration of albumin nanoparticles suspension intravenously

that maximum serum availability of $425 \mathrm{ng} / \mathrm{ml}$ of curcumin was observed in case of albumin nanoparticles and in case of free curcumin only about $276 \mathrm{ng} / \mathrm{ml}$ of curcumin was observed. The drug was detectable for only 24 hours and resulted in the known pharmacokinetic pattern of curcumin. Furthermore, it was observed that unlike free curcumin, curcumin from nanoparticles was detected for a longer period of time of about 25 days when observed for about 30 days after administration [Figure 3]. Our result suggested the slow release of curcumin from nanoparticles increased the bioavailability of delivered curcumin with time as observed for about 30 days of our observation. This was further corroborated with the tissue uptake. In this case, same dose of the drug over a period of 30 days that was equivalently present in the nanoparticles administered was administered. In pharmacokinetic tissue distribution of drug from nanoparticulate formulation, curcumin concentration in lungs and brain was significantly higher [Table 3] when compared to the repeated i.v. administration. These are the common sites of metastases in breast cancer. Though not investigated here, the above observations suggest that curcumin nanoparticles fabricated here could be highly effective against breast cancer metastases. ${ }^{[12]}$

The antiproliferation efficacy of free curcumin and nanocurcumin on breast cancer cell line (MDA-MB-231) was determined at different concentrations $0,20,40,60,80,100,120 \mathrm{mcM}$ for about $72 \mathrm{hrs}$. The results showed that nanocurcumin was more anti proliferative than that of free curcumin [Figure 4] and this could be mainly attributed to the higher intracellular drug levels with the drug-loaded nanoparticles.

The results of the study thus revealed that nanoparticular formulation developed here will be a suitable delivery system for curcumin to treat breast cancer. The study although corroborating previous results puts forward the data for the practical application

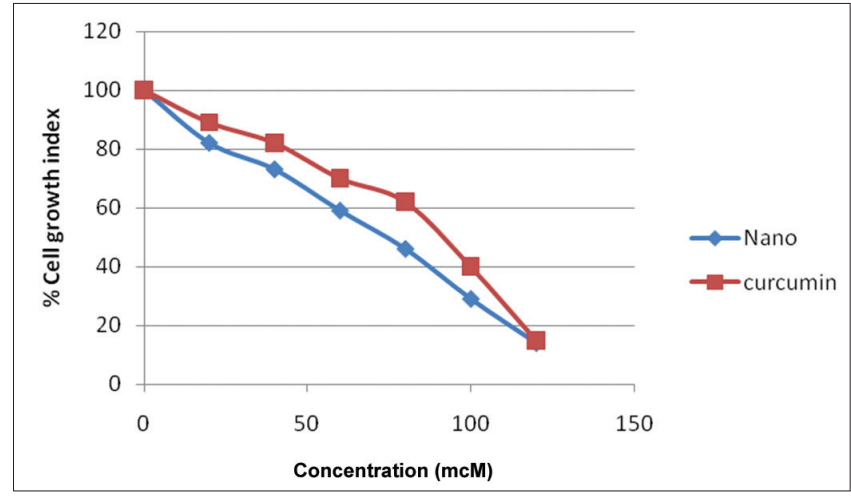

Figure 4: Percent cell growth index of curcumin and nanocurcumin in breast cancer cell lines

of nanotechnology to cover curcumin in its perview for bringing it into the forefront of the fight against cancer. The overall results justified that nanoparticulate curcumin hold better chemotherapeutic properties in breast cancer than free curcumin due to its better bioavailability, sustained, and targeted release and better antiproliferative activity in tumor cells.

\section{ACKNOWLEDGEMENT}

The authors of the manuscript would acknowledge the management of Mother Teresa College of Pharmacy for providing support during preparation of the manuscript. The authors would like to acknowledge the management of Vaagdevi College of Pharmacy, Warangal, for providing facilities for the conduction of the work. One of the Authors, Dr. Aukunuru Jithan would like to acknowledge the Department of Science and Technology (DST), Government of India. This project is partly funded under a SERC-DST Fast Track Proposal for Young Scientists awarded to Dr. Aukunuru Jithan.

\section{REFERENCES}

1. Aggarwal BB, Sung B. Pharmacological basis for the role of curcumin in chronic diseases: An age-old spice with modern targets. Trends Pharmacol Sci 2009;30:85-94.

2. Kunnumakkara $A B$, Anand P, Aggarwal BB. Curcumin inhibits proliferation, invasion, angiogenesis and metastasis of different cancers through interaction with multiple cell signaling proteins. Cancer Lett 2008;269:199-225.

3. Anand P, Sundaram C, Jhurani S, Kunnumakkara AB, Aggarwal BB. Curcumin and cancer: An "old-age" disease with an "ageold" solution. Cancer Lett 2008;267:133-64.

4. Sharma RA, McLelland HR, Hill KA, Ireson CR, Euden SA, Manson MN, et al. Pharmacodynamic and pharmacokinetic study of oral Curcuma extract in patients with colorectal cancer. 
Clin Cancer Res 2001;7:1894-900.

5. Ray B, Bisht S, Maitra A, Maitra A, Lahiri DK. Neuroprotective and neurorescue effects of a novel polymeric nanoparticle formulation of curcumin (NanoCurc ${ }^{\mathrm{TM}}$ ) in the neuronal cell culture and animal model: Implications for Alzheimers disease. J Alzheimers Dis 2011;23:61-77.

6. Shehzad A, Wahid F, Lee YS. Curcumin in cancer chemoprevention: Molecular targets, pharmacokinetics, bioavailability, and clinical trials. Arch Pharm (Weinheim) 2010;343:489-99.

7. Bisht S, Feldmann G, Soni S, Ravi R, Karikar C, Maitra A. Polymeric nanoparticle-encapsulated curcumin ("nanocurcumin"): A novel strategy for human cancer therapy. J Nanobiotechnology 2007;5:3.

8. Viswanath G, Jithan A, Reddy VM. Development of new delivery strategies to increase bioavailability of curcumin. Int J Pharm Sci Nanotechnol 2009;1:335-40.

9. Nattha K, Sanae K, Amaravadee J, Daungkhae M, Thitima C, Pharkphoom P. Increased solubility, dissolution and physicochemical studies of curcumin-polyvinylpyrrolidone K-30 solid dispersions. World Acad Sci Eng Technol 2009;55:229-34.

10. Yadav VR, Suresh S, Devi K, Yadav S. Effect of cyclodextrin complexation of curcumin on its solubility and antiangiogenic and anti-inflammatory activity in rat colitis model. AAPS PharmSciTech 2009;10:752-62.

11. Shaikh J, Ankola DD, Beniwal V, Singh D, Kumar MN. Nanoparticle encapsulation improves oral bioavailability of curcumin by at least 9-fold when compared to curcumin administered with piperine as absorption enhancer. Eur J Pharm Sci 2009;37:223-30.

12. Shahani K, Swaminathan SK, Freeman D, Blum A, Ma L, Panyam J. InjecTable sustained release microparticles of curcumin: A new concept for cancer chemoprevention. Cancer Res 2010;70:4443-52.

13. Sou K, Inenaga S, Takeoka S, Tsuchida E. Loading of curcumin into macrophages using lipid-based nanoparticles. Int J Pharm 352:287-93.

14. Li L, Ahmed B, Mehta K, Kurzrock R. Liposomal curcumin with and without oxaliplatin: Effects on cell growth, apoptosis, and angiogenesis in colorectal cancer. Mol Cancer Ther 2007;6:1276-82.

15. Vemula PK, Li J, John G. Enzyme catalysis: Tool to make and break amygdalin hydrogelators from renewable resources: A delivery model for hydrophobic drugs. J Am Chem Soc 2006;128:8932-8.

16. Mohanty C, Sahoo SK. The in vitro stability and in vivo pharmacokinetics of curcumin prepared as an aqueous nanoparticulate formulation. Biomaterials 2010;31:6597-611.

17. Kim TH, Jiang HH, Youn YS, Park CW, Tak KK, Lee S, et al. Preparation and characterization of water-soluble albuminbound curcumin nanoparticles with improved antitumor activity. Int J Pharm 2011;403:285-91.

18. Das S, Benerjee R, Bellare J. Aspirin loaded albumin nanoparticles by coacervation: Implications in drug delivery. Trends Biomakers Artif Organs 2005;18:203-12.

19. Bourassa P, Kanakis CD, Tarantilis P, Pollissiou MG, TajmirRiahi HA. Resveratrol, genistein, and curcumin bind bovine serum albumin. J Phys Chem B 2010;114:3348-54.

20. Wartlick H, Spankuch-Schmitt B, Strebhardt K, Kreuter J, Langer $\mathrm{K}$. Tumor cell delivery of antisense oligonucleotides by human serum albumin nanoparticles. J Control Release 2004;96:48395.

21. Mirtsching B, Cosgriff T, Harker G, Keaton M, Chidiac T, Min M. A Phase II study of weekly nanoparticle albumin-bound paclitaxel with or without transtuzumab in metastatic breast cancer. Clin Breast Cancer 2011;11:121-8.

22. Weber C, Kreuter J, Langer K. Desolvation process and surface characteristics of HAS-nanoparticles. Int $\mathrm{J}$ Pharm 2000;196:197-200.

23. Suresh K, Hemanth Kumar Y, Bonepally CR, Pradeep Kumar Y, Jithan A. Liposomal delivery of curcumin to liver. Turk J Pharm Sci 2010;7:89-98

Source of Support: SERC-DST , Conflict of Interest: None declared.

Received: 17-02-11, Revised: 25-02-11, Accepted: 02-03-11

\section{Staying in touch with the journal}

1) Table of Contents (TOC) email alert

Receive an email alert containing the TOC when a new complete issue of the journal is made available online. To register for TOC alerts go to www.jpionline.org/signup.asp.

2) RSS feeds

Really Simple Syndication (RSS) helps you to get alerts on new publication right on your desktop without going to the journal's website. You need a software (e.g. RSSReader, Feed Demon, FeedReader, My Yahoo!, NewsGator and NewzCrawler) to get advantage of this tool. RSS feeds can also be read through FireFox or Microsoft Outlook 2007. Once any of these small (and mostly free) software is installed, add www.jpionline.org/rssfeed.asp as one of the feeds. 